\title{
Lung cancer screening: Is there a future?
}

Mary ER O'Brien

Department of Medicine, The Royal Marsden NHS Foundation Trust, Sutton, Surrey, UK
Address for correspondence: Mary ER O'Brien

Department of Medicine, The Royal

Marsden NHS Foundation Trust,

Sutton, Surrey, UK

E-mail: mary.obrien@rmh.nhs.uk

\section{A B S T R A C T}

Lung cancer is the leading cause of cancer death worldwide with an average rate of 40-100/100,000 depending on the level of deprivation, and the rates are higher in smokers. The National Lung Screening Trial using three consecutive annual lowdose computed tomography scans is the first and largest screening study to show clear evidence of a significant reduction in lung cancer mortality in selected high-risk subjects. The many on-going European screening studies will generate information on the groups of subjects that may or may not benefit from screening (demographics, pack-years smoked, length of smoking, number of years from quitting etc.) and the required frequency and duration of the intervention. Smoking cessation remains the most important tool for general improvement in health outcomes and in particular lung cancer prevention. Early intervention for investigations of symptoms that are considered mild or common could also change the outcome. Doctors and patients must become increasingly aware that these common symptoms are also potentially symptoms of lung cancer and are not 'normal' even in smokers.

Key words: Lung cancer, low dose computed tomography scan, screening

\section{INTRODUCTION}

Worldwide approximately 14.2 million cases of cancer were diagnosed in 2012, and there were 8.2 million deaths from cancer in that year. Lung cancer accounts for 1.82 million of these cases, but 1.6 million deaths (19.5\%). ${ }^{[1]}$ In Europe, more than 410,000 new cases of lung cancer were estimated to have been diagnosed in 2012, approximately $10 \%$ of these were in the UK. ${ }^{[2]} 70 \%$ of these patients will have died by the end of the next year.

In Globoscan 2012, lung cancer was the $4^{\text {th }}$ most frequent cancer in India in its population of just over a billion and accounted for about 1 million cases per year again most of these of lung cancer died within the $1^{\text {st }}$ year. Cervix/uterine, breast and lip/oral cavity were first to third respectively. Incidence and mortality rates are influenced by the quality and completeness of the data, but lung cancer incidence is also dependent on population demographics and development indices. ${ }^{[1]}$ This would suggest that the first priority in cancer prevention or early detection in many countries, including India, should

\begin{tabular}{|l|l|}
\hline \multicolumn{3}{c|}{ Access this article online } \\
\hline Quick Response Code: & Website: \\
\hline & www.jmpo.org \\
\hline
\end{tabular}

be women' health, and the second should be related to tobacco control.

Advances in treatment of cancer have only had a small influence on survival over the past 30 years. The vast majority of patients are diagnosed at a symptomatic, advanced and incurable stage as early stage, potentially curable lung cancer is usually asymptomatic. Screening for lung cancer has been examined with a variety of strategies for many years, without a clear mortality benefit emerging. The earliest screening studies involved chest radiography (chest X-ray [CXR]) and/or sputum cytology. These negative studies did not lead to a screening program.

The first screening study to demonstrate a mortality benefit was the National Lung Screening Trial (NLST) in the USA. ${ }^{[3]}$ The NLST recruited 53,454 male and female subjects, aged between 55 and 74 years, at high risk of lung cancer, defined as a 30 pack-years or more smoking history. Subjects were either current smokers or former smokers who had quit within the previous 15 years. Subjects were randomly assigned to low-dose spiral computed tomography (LDCT) scans or to CXR at baseline and annually for 2 years (total three scans) and then followed-up for 5 years. The incidence of lung cancer was 645 cases per 100,000 person-years (1060 cancers over the 5 year study period) in the LDCT group, when compared with 572 cases per 100,000 person-years (941 cancers) in the CXR group. Importantly, more early stage (stage IA and IB) operable lung cancers were seen in the LDCT group than in the CXR group at diagnosis; $50 \%$ 
and $31 \%$ respectively which is the 'stage shift' that most people feel is necessary to change the course of lung cancer and decrease lung cancer mortality rates. There were fewer advanced cancers (stage IV) in the LDCT group than in the CXR group; $22 \%$ and $36 \%$ respectively. This study reached the primary end point of a $20 \%$ reduction in lung cancer mortality in the LDCT screened group ( $95 \%$ confidence interval [CT]: 6.8-26.7; $P=0.004$ ) compared to the CXR group. This was based on 356 deaths out of 1060 detected lung cancers in the LDCT group, and 443 deaths out of 941 detected lung cancers in the CXR group, corresponding to rates of death from lung cancer of 247 per 100,000 personyears in the LDCT group and 309 per 100,000 person-years in the CXR group.

The number needed to screen with LDCT to prevent one lung cancer death was 320. All cause mortality, the secondary endpoint, showed a 6.7\% (95\% CI: 1.2-13.6, $P=0.02)$ reduction in the LDCT group. At all three rounds of screening, the rate of further diagnostic workup resulting from the detection of small asymptomatic pulmonary nodules was higher in the LDCT group, as was the detection of clinically significant findings other than lung cancer $(7.5 \%$ vs. $2.1 \%)$, respectively.

This NLST with 53,454 subjects is greater than the accrual for all of the European studies combined. Therefore data from the NLST are likely to contribute significantly to any future meta-analyses. The screening approaches taken by the European studies are diverse, and they will be important to answer questions on the management of positive scans, the role of positron emission tomography (PET) scanning, etc. However, individually any one of them is unlikely to give the same $20 \%$ reduction in lung cancer mortality reported by the NLST due to their individual smaller size and different patient selection criteria in addition to different scanning frequency. If these studies are indeed positive it will make the issue of screening more urgent but if they are negative then we are still left with the NLST data and its provocative positive finding. This commentary aims to put the data in the perspective of current lung cancer treatment and outcomes.

\section{IMPLICATIONS FOR NATIONAL SCREENING PROGRAMMES}

As the results of the NLST would suggest 67,000 lives could be saved each year on an incidence of 410,000 cases in Europe, each country needs to compare itself to this figure and assess what would be the approximate number of lives saved and the value to the population overall.

The vast majority of people who take part in any screening program will not have lung cancer. Across all 3 rounds of the NLST imaging procedures, $24.1 \%$ of LDCT scans were positive - the scan showed something that was not considered normal and further investigation or earlier follow-up was needed; following further evaluation, most of these patients did not have lung cancer. Lung cancer was only confirmed in $3.6 \%$ of the positive scans in the LDCT group representing $0.87 \%$ of all LDCT scans. ${ }^{[3]}$

\section{WHAT IS THE DEFINITION OF A POSITIVE SCAN?}

Rates of lung nodules and abnormalities vary in populations and are largely dictated by other community disease, in particular previous TB in many parts of the world and ongoing chronic obstructive pulmonary disease (COPD). In the NLST trial, a noncalcified nodule $>4 \mathrm{~mm}$ was defined as positive and triggered further investigations. Overall approximately $75 \%$ of scans were normal, the false positive rate (abnormality requiring further investigation, which was later shown to not be lung cancer) was around $24 \%$ with LDCT and the true lung cancer positivity rate was $0.87 \%$. The Dutch-Belgium NELSON study has reported that the frequency of positive results, leading to further investigation, can be markedly reduced using a more restrictive size definition.

The NELSON study is the largest screening study from Europe. Eligible subjects in this study were male subjects aged 55-75 who were current or former smokers (quit $<10$ years ago) who smoked $>15$ cigarettes/day for $>25$ years or $>10$ cigarettes/day for $>30$ years, that is, 18-15 pack years (NLST used entry criteria of 30 pack years and having stopped $<15$ years). Subjects were randomized to LDCT screening (baseline, year 1 and year 3) or to no screening. In the NELSON study, a scan was considered positive if the volume of a noncalcified nodule was $>500 \mathrm{~mm}^{3}$ (approximately $10 \mathrm{~mm}$ in one diameter). A scan was considered negative if the volume of the nodule was $<50 \mathrm{~mm}^{3}$ (approximately $5 \mathrm{~mm}$ diameter). A scan was labeled as indeterminate if the volume of the nodule was between 50 and $500 \mathrm{~mm}^{3}$. In subjects with an indeterminate result, follow-up criteria were defined for the study according to doubling time using radiological threedimensional CT measurements. With these stricter criteria, the positivity rate and thus intervention rate went down compared to the NLST from $24 \%$ to a reasonable $2.6 \%$, but still $64 \%$ of these were false positive. ${ }^{[4]}$ PET-LDCT is part of the Italian MILD study. ${ }^{[5]}$

\section{WHO WOULD BENEFIT MOST FROM SCREENING?}

A national screening program must be synergistic with the public health message of smoking cessation, and an effective screening tool must not weaken this message, 
though more publicity could increase awareness and increase smoking cessation — current data supports neither hypothesis.

No screening program is without risks to the individual when false positives and postimaging investigational procedures are taken into account. The detrimental effect on quality of life is well known for breast cancer screening with mammography, but it is short term., More will be reported for lung cancer screening from the NELSON trial. ${ }^{[4]}$ Sixteen subjects in the LDCT group of the NLST died within 60 days of having an invasive diagnostic procedure; six of whom did not have lung cancer. The corresponding figure for the CXR group was 10; all of whom had lung cancer. ${ }^{[3]}$

Like the NLST, the European LDCT screening studies have defined the risk groups based on smoking status and age, yet, despite screening "high risk" subjects, most studies have reported a baseline cancer prevalence of only 1-3\%. The UK Lung Screening trial (UKLS) aimed for a population with a predicted $5 \%$ prevalence rate of lung cancer and employed a validated prediction model, the Liverpool Lung Project (LLP) Risk Prediction Model, to include high risk subjects for randomization to a once only CT scan or no CT screening scan. The LLP risk model divides patients into high and low risk groups — the high risk being all smokers with at least a 30 pack year history, nonsmokers for $<10$ years with a 30 pack year history and at least one other prognostic factor (e.g., previous history of any cancer, family history of lung cancer in first degree relative, a history of pneumonia or a prexisting diagnosis of COPD or dust/asbestos exposure) were also included. The study is closed at 4,000 randomized patients and should report some data in 2015. ${ }^{[6]}$

The role of COPD as a risk factor for lung cancer is not as clear as one would think, despite the common risk factor of smoking. The Lung-SEARCH trial is a randomized study of surveillance for lung cancer in subjects with COPD. In this study, subjects in the intervention group had annual sputum tests followed by LDCT and fluorescence bronchoscopy if the sputum was abnormal. The study is closed, and results are awaited (Lung-SEARCH UKCRN ID 2225).

\section{WHAT IS THE OPTIMAL INTERVAL BETWEEN SCREENING SCANS AND HOW MANY SCREENING ROUNDS ARE REOUIRED?}

The yearly detection rates from the NLST shows a surprising constant rate of detection $(0.68-1.0 \%$ /year) suggesting that continued screening beyond 3 years might be useful. A trial by Henschke et al. compared lung cancer mortality in a cohort of 7,995 smokers who underwent LDCT screening for lung cancer in New York State (NYS) with two unscreened cohorts. ${ }^{[7]}$ The mortality reduction due to screening in NYS cohort becomes evident in the $4^{\text {th }}$ year and even more evident in the $6^{\text {th }}$ through $8^{\text {th }}$ years. When limited rounds of screening were provided (after the 8 years) deaths in the NYS cohort increased.

This also suggests that the benefit seen in the NLST trial may be an underestimation as in the protocol they stopped scanning at 3 years.

In the Multicenter Italian Lung Detection (MILD) trial, subjects were randomized to LDCT or usual care for 10 years. In the LDCT group, a second randomization compares LDCT screening yearly versus 2 -yearly. ${ }^{[5]}$ The 5 -year data from the MILD study demonstrated similar LDCT detected lung cancer diagnosis rates and mortality between both yearly and 2-yearly screening groups, although numbers are small $(n=2,376)$. The UKLS will give information on the utility of a single random LDCT scan. ${ }^{[6]}$

\section{ALTERNATIVES TO LOW DOSE SPIRAL COMPUTED TOMOGRAPHY SCREENING}

Applying technology is often easier than changing human behavior. Tobacco control would largely solve the lung cancer problem. Unlike breast cancer screening with mammograms, the proposed technology for lung cancer is expensive and the population at risk older and in general a less compliant group.

Given all the caveats, there is a desperate need to look at other technologies. Apart from refinement and better definition of the highest risk group to screen, digital CXRs, fluorescent bronchoscopy and less frequent scanning will all need to go through the rigors of another 5-10 years of clinical trials. Adding lung cancer screening to TB screening, cardiac check-ups and blood tests fishing for tumor antigen panels are all potential ways of indirectly addressing the problem. For lung cancer and oral cavity cancers (highest incidence in male Indians) we have access to the at risk target tissue in the mucosa and bronchial lining, through the mouth and airways. Screening programs for the bowel and tissue of the cervix have been able to make use of this. Real progress with a simple screening test and screening program has now been developed for colon cancer by the search for fecal occult blood and now tumor DNA in the excreta. ${ }^{[8]}$ In India, an alternative to Pap smear screening for cervical cancer has been developed using the application of acetic acid to the cervix and visually assessing the result. ${ }^{[9]}$ 
We need our researchers to look at what is happening in other cancers and aggressively try and give us a solution for at risk smokers or ex-smokers.

\section{ALTERNATIVES TO LOW DOSE SPIRAL COMPUTED TOMOGRAPHY SCREENING WHILE WE ARE WAITING}

Taking a hard look at the data - maybe LDCT screening is not indeed the best way to go within the next 5 years so clinicians must have a different strategy. Not only is lung cancer preventable but it is currently diagnosed late at an advanced, noncurable stage, as patients and health professionals have no strategy to diagnose early.

A campaign urging people with a 3 week old cough to get checked out by their doctor has resulted in a rise in the number of lung cancers detected earlier according to an analysis of the results of a local CRUK uncontrolled initiative in the UK-around 700 lung cancers were diagnosed - many of these at an early stage in this program in a geographic area that would have predicted 400 cancers in the same time period. ${ }^{[1]}$ In our own practice, we have found that community pharmacists were very good at interacting with patients and highlighting symptoms that led to a diagnosis of airways diseases in an unsuspecting small cohort. ${ }^{[10]}$ The involvement of community health professionals in a proactive early symptom detection program is an approach we do have access to and could develop and assess.

However, our patients who are seen in the clinics with advanced stage lung cancer and who have never smoked or have stopped smoking for $>15$ years, would not have been candidates for any of these trials or screening interventions. In parallel to imaging studies, the search must continue for a germline signal or a biomarker for this disease that may be of use in populations irrespective of smoking history.

\section{CONCLUSION}

The NLST is the first positive screening trial for the detection of lung cancer. European screening trials will report in the next few years with additional data on the frequency of intervention and subject selection. A nonimaging gene or biomarker is needed in parallel to imaging.
National lung cancer screening programs need to be cost-effective and run in parallel with smoking cessation programs.

\section{ACKNOWLEDGMENTS}

I acknowledge NHS funding to the Royal Marsden Hospital/ Institute of Cancer Research NIHR Biomedical Research Centre.

\section{REFERENCES}

1. Ferlay J, Soerjomataram I, Dikshit R, Eser S, Mathers C, Rebelo $\mathrm{M}$, et al. Cancer incidence and mortality worldwide: Sources, methods and major patterns in GLOBOCAN 2012. Int J Cancer 2014.

2. Available from: http://www.cancerresearchuk.org/cancerinfo/cancerstats/world/. [Last accessed on 2014 Sep].

3. National Lung Screening Trial Research Team, Aberle DR, Adams AM, Berg CD, Black WC, Clapp JD, et al. Reduced lung-cancer mortality with low-dose computed tomographic screening. N Engl J Med 2011;365:395-409.

4. van Klaveren RJ, Oudkerk $M$, Prokop $M$, Scholten ET, Nackaerts K, Vernhout $\mathrm{R}$, et al. Management of lung nodules detected by volume CT scanning. N Engl J Med 2009;361:2221-9.

5. Pastorino U, Rossi M, Rosato V, Marchianò A, Sverzellati N, Morosi $\mathrm{C}$, et al. Annual or biennial CT screening versus observation in heavy smokers: 5-year results of the MILD trial. Eur J Cancer Prev 2012;21:308-15.

6. Baldwin DR, Duffy SW, Wald NJ, Page R, Hansell DM, Field JK. UK Lung Screen (UKLS) nodule management protocol: Modelling of a single screen randomised controlled trial of low-dose CT screening for lung cancer. Thorax 2011;66:308-13.

7. Henschke Cl, Boffetta P, Gorlova O, Yip R, Delancey JO, Foy M. Assessment of lung-cancer mortality reduction from CT Screening. Lung Cancer 2011;71:328-32.

8. Anderson JC, Shaw RD. Update on colon cancer screening: Recent advances and observations in colorectal cancer screening. Curr Gastroenterol Rep 2014;16:403.

9. Shastri SS, Mittra I, Mishra GA, Gupta S, Dikshit R, Singh S, et al. Effect of VIA screening by primary health workers: Randomized controlled study in Mumbai, India. J Natl Cancer Inst 2014;106:dju009.

10. Punwani R, Draper A, Loke T, Keddie Z, O'Brien M. Community pharmacy referrals project: Increasing awareness and early diagnosis of respiratory disease via a direct pathway to secondary care. Lung Cancer 2014;83 Suppl 1: S31-2.

How to cite this article: O'Brien ME. Lung cancer screening: Is there a future?. Indian J Med Paediatr Oncol 2014;35:249-52. Source of Support: Nil, Conflict of Interest: None declared. 